\title{
Les randonnées (contes énumératifs) - Entre récits, virelangues et facéties
}

\author{
Michèle Simonsen \\ Københavns Universitet, Copenhague \\ simonsen.michele@gmail.com
}

\section{RÉSUMÉ}

Je vous propose de nous interroger sur la nature et le statut des randonnées-contes énumératifs-à partir du conte Wee Wee Woman (ATU 20I6). Ce conte exemplifie les difficultés que soulève la classification des randonnées. Les catalogues AT et ATU semblent utiliser la répétition du mot « tout petit » comme seul critère de distinction. Or la description qu'ils donnent de ce conte-type ne s'applique qu'à certaines des versions connues.

A la différence de certaines randonnées comme The Goat who would not Go Home ATU 20I5, le conte Wee wee Woman ne peut pas être relié à quelque usage religieux ou rituel, et il est peu probable qu'il ait jamais été utilisé à des fins didactiques. Sous ses formes les plus simples, c'est presque un conte d'attrape, qui frustre l'auditeur dans son attente d'un vrai récit complet. On peut y voir une parodie de conte, l'exploration facétieuse des limites de la narratologie. Jusqu'où peut-on pousser la simplicité d'un énoncé, et la trivialité de ses détails, avant qu'il cesse d'être un récit? Par ailleurs, cette randonnée, plus que d'autres peut-être, a un fort élément de virelangue ( tongue-twister»).

Plus que tous les autres types de contes, les randonnées ne prennent tout leur sens que dans une performance orale.

\section{MOTS CLÉS}

randonné, ATU 20I6, ATU 20I5, virelangue, performance orale

RESUM

Us proposo que ens preguntem sobre la naturalesa i l'estatus dels les rondalles enumeratives a partir de la rondalla Wee Wee Woman (ATU 20I6). Aquesta rondalla exemplifica les dificultats que planteja la classificació de les rondalles enumeratives. Els catàlegs AT i ATU semblen utilitzar la repetició del mot «diminut» com a únic criteri de diferenciació. La descripció, però, que donen d'aquesta rondalla-tipus només s'aplica a algunes de les versions conegudes.

A diferència d'algunes rondalles enumeratives com ara The Goat Who Would Not Go Home (ATU 20I5), el conte Wee Wee Woman no es pot pas relacionar amb cap ús 
religiós o ritual, i és poc probable que mai s’hagi utilitzat amb fins didàctics. En les formes més simples, és gairebé una rondalla-trampa, que frustra l'auditori en l'expectativa d'un autèntic relat complet. S'hi pot veure una paròdia de rondalla, l'exploració graciosa dels limits de la narratologia. Fins on podem exagerar la simplicitat d'un enunciat, i la trivialitat dels seus detalls, abans que deixi de ser una història? D'altra banda, aquesta rondalla enumerativa, potser més que no d'altres, té un fort element d'embarbussament (tongue-twister).

Més que qualsevol altra tipus d'històries, les rondalles enumeratives prenen sentit en una actuació oral.

\title{
PARAULES CLAU
}

rondalla enumerativa, ATU 20I6, ATU 20I5, embarbussament, actuació oral

\begin{abstract}
I would like to take the tale of the Wee Wee Woman (ATU 20I6) as a starting point for reflecting on the nature and status of chains based on number. This tale exemplifies the difficulties that arise when classifying chains. The AT and ATU catalogues seem to use the repetition of a tiny word as the sole differentiating criterion. However, the description that they give of this tale-type can only be applied to certain known versions.

In contrast to certain chains such as The Goat Who Would Not Go Home ATU 20I5, the tale of the Wee Wee Woman cannot be related to any religious practice or ritual, and it is unlikely that it has ever been used for didactic purposes. In its simplest form, it makes fun of its listeners by confounding their expectations of hearing a full tale. It is a parody of a tale; a witty exploration of the limits of narrative. To what extent can we exaggerate the simplicity of a tale and the trivial nature of its details before it ceases to be a story? Furthermore, this chain perhaps more than others makes plentiful use of the tongue-twister.

More than any other type of stories, cumulative tales have sense in an oral performance.
\end{abstract}

\section{KEYWORDS}

cumulative tales, ATU 20I6, ATU 20I5, tongue-twister, oral performance 


\section{Introduction}

J'ai pris pour sujet de réflexion le genre assez peu étudié des « randonnées »— c'est ainsi que les folkloristes français désignent généralement les contes énumératifs, «cumulative tales» en anglais.

Si l'on voulait donner une définition de ces randonnées, ce serait quelque chose comme: conte énumératif, court, avec un enchaînement de situations, d'éléments ou de personnages qui se répètent jusqu'au dénouement.

L'exemple le plus connu est sans doute celui de la chèvre qui ne veut pas sortir du champ, (ATU 2015 The Goat who would not go Home), que vous connaissez probablement tous sous une forme ou sous une autre dans vos répertoires nationaux, et qui existe aussi souvent sous forme de chanson.

Voici un exemple un peu moins connu, adapté du folklore danois: Le Chat ventru: (AT 2027 The Fat Cat, ATU 2028, The devouring Animal That Was Cut Open.)

Il était une fois une petite vieille, qui habitait seule avec son chat, un petit chat très très gourmand mais pas toujours obéissant.

Voilà qu'un jour elle fait cuire de la bouillie de gruau. En attendant qu'elle refroidisse, elle veut aller faire des fagots. Mais d'abord, elle sert une bonne écuelle de bouillie à son chat.

«Voilà du gruau, mange le tien, mais surtout ne touche pas au mien! »

«Miaou! » répond le chat, et la petite vieille s'en va dans la forêt.

Aussitôt, le chat se jette sur son gruau. Il a vite fait de lécher l'écuelle! Et comme il a encore très faim, il mange aussi le gruau de la vieille.

Mais ça ne lui suffit pas, non! Alors vite, il avale aussi la marmite et la cuiller à pot.

La petite vieille rentre bientôt avec son fagot. Vite, elle fait un bon feu. Oh! comme elle va se régaler de gruau, les pieds et le dos bien au chaud!

Mais au fait, où est passé le gruau?

Elle cherche partout, sur le poêle, dans la huche, dans tous les recoins de la maison. Mais le gruau a disparu.

«Dis-moi, mon petit chat, sais-tu où sont passés mon gruau, ma marmite et ma cuiller à pot? »

«Mais oui » dit le chat, « je les ai mangés, et maintenant je vais te manger toi aussi. »

Et hop! Il avale la petite vieille, et puis il s'en va par les grands chemins.

Peu après, il rencontre une pie jacasse. La pie jacasse se tracasse:

«Oh! le gros ventre! Mais qu'est ce que tu as donc mangé, mon petit chat, pour être si ventru?»

« On serait ventru à moins! », répond le chat. «J'ai mangé tout le gruau, la marmite et la cuiller à pot, la petite vieille avec, et maintenant je vais te manger toi aussi! »

Et hop! Il avale la pie jacasse et continue son chemin.

Mais il a encore très faim! 
Peu après, il rencontre deux oies sauvages en plein vol. Les deux oies s'affolent:

«Oh! le gros ventre! Mais qu'est-ce que tu as donc mangé, mon petit chat, pour être si ventru?»

« On serait ventru à moins! J'ai mangé tout le gruau, la marmite et la cuiller à pot, la vieille et la pie jacasse, et maintenant je vais vous manger vous aussi! »

Et hop! Le chat avale les deux oies en plein vol et continue son chemin.

Mais il a encore très faim!

Un peu plus loin il rencontre trois demoiselles en dentelles. Les demoiselles l'interpellent:

«Oh! le gros ventre! Mais qu'est-ce que tu as donc mangé, mon petit chat, pour être si ventru?»

« On serait ventru à moins! », répond le chat. « J'ai mangé tout le gruau, la marmite et la cuiller à pot, la vieille et la pie jacasse, deux oies sauvages en plein vol, et maintenant je vais vous manger vous aussi! »

Et hop! Le chat avale les trois demoiselles en dentelles et continue son chemin.

Mais il a encore très faim!

Un peu plus loin, il rencontre quatre dames en crinoline. La plus belle des quatre s'écrie:

«Oh! le gros ventre! Mais qu'est-ce que tu as donc mangé, mon petit chat, pour être si ventru?»

«On serait ventru à moins! J'ai mangé tout le gruau, la marmite et la cuiller à pot, la vieille et la pie jacasse, deux oies sauvages en plein vol, trois demoiselles en dentelles, et maintenant je vais vous manger vous aussi! » Et hop! Le chat avale les quatre dames en crinoline et continue son che$\min$.

Mais il a encore très faim!

Peu après, voilà qu'il rencontre un bûcheron avec sa cognée.

Le bûcheron fait les yeux ronds:

«Oh! le gros ventre! Mais qu'est-ce que tu as donc mangé, mon petit chat, pour être si ventru?»

« On serait ventru à moins! J'ai mangé tout le gruau, la marmite et la cuiller à pot, la vieille et la pie jacasse, deux oies sauvages en plein vol, trois demoiselles en dentelles, quatre dames en crinoline, et maintenant je vais te manger to aussi»

«Ah! Tu crois ça? », dit le bûcheron. «C'est ce qu'on va voir! »

Et d'un grand coup de cognée, il fend le chat en deux morceaux.

Et il en sort aussitôt:

1 es belles dames en crinoline

les demoiselles en dentelles 
les deux oies sauvages

la pie jacasse

et la petite vieille,

qui remet le gruau dans la marmite,

reprend sa cuiller à pot,

et rentre chez elle à pied.

\section{Classification des randonnées}

Ces randonnées constituent le plus gros de la classe des contes formulaires («formular tales ») ou dans la classification AT/ATU. ${ }^{\mathrm{r}}$

Cette classe de contes formulaires, se situe au premier degré de classification de tous les contes populaires, c'est-à-dire au même niveau d'arborescence que I. Les CONTES D’ANIMAUX («Animal Tales »), II. Les CONTES PROPREMENT DITS, III. Les CONTES FACETIEUX ( «Jokes and anecdotes»), V. Les CONTES NON CLASSES. C'est donc un groupe important. Je ne parle pas ici du nombre de contes-types et de variantes ni de la popularité de ces contes formulaires, mais de leur importance structurale. Pour AT et pour ATU, les contes formulaires ont une spécificité très nette.

Dans l'ATU, cette classe de contes formulaires comprend trois sous-classes: les randonnées ( « cumulative tales » entre 2000 à 2075), puis les contes d'attrape (de 2200 à 2299), et enfin une sous-classe de résidus, intitulés « autres contes formulaires » (2300 à 2399). Les numéros entre 2075 et 2I99 ne sont pas attribués. Il ne faudrait pourtant pas en conclure que ces trois sous-classes de contes formulaires sont aussi fournies les unes que les autres et comptent 99 contes-types chacune. En effet, la numérotation n'est pas linéaire: il y a beaucoup de numéros non attribués. Sont répertoriés:

- IO contes d'attrape-types: 2200, 2202, 2224, 2205, 2250, 225I, 2260, 227I, $2275,2280$.

- 9 autres contes formulaires-types: 2300, 230I, 2302, 2335, 2340, 2400, 240I, 2404, 24II.

- Et une soixantaine de randonnées-types:2 2009, 20IO, 20IOA, 20IO B, 20IOI, 2OIOIA, 2OII, 2OI2, 2012 A-D (See Type 2OI2), 2OI3, 2OI4, 2014 A (See Type 2OI4), 20I5, 20I5* (See Type 2030) 20I6, 2OI7, (See 2302) 2018 (See 2043) 2OI9, 2OI9*, 2O2I, 2O2I A (See Type 2O2I), 2O2I B, 2O2I* (See Type 2022), 2022A (See Type 2022), 2022, 2022A (See 2022), 2022 B, 2O23, 2O24*, 2O25, 2027, $2 \mathrm{O} 27$ A, 2O28, 2028 A (See 2O28), 2O3O, 2O3OA-J (See 203O), 203OA*-2O3OE*, 2O3I, 2O3I A, 2O3I B, 203I C, 2032, 2032A (See 2032), 2033, 2034, 2034A (See 295), 2034 B, 2034C, 2034 E, 2034 F, 2034A* $2035,2036,2037 A^{*}, 2039,2040,204 \mathrm{I}, 2042,2042 A^{*}, 2042 B^{*}-D^{*}$ (See 2042A*), 2043, 2044, 2075 (See Type I06).

I. AT: cf. Aarne-Thompson (I96I); ATU: cf. Uther (2004).

2. Le nombre varie un peu selon que l'on tient compte des renvois à un autre conte-type, et des sous-classes A, B, C, D. A cet égard aussi l'ATU a simplifié l'AT. 
Les randonnées se taillent donc la part du lion dans la classe des contes formulaires.

Il existe plusieurs systèmes de classification des randonnées:

I) Archer Taylor (I933) ${ }^{3}$ et Aarne-Thompson (I96I) distingue les sous-groupes suivants:

- chaînes fondées sur des nombres ou des objets, comme AT 2009 Origin of Chess ou AT 2oro Ehod mi yodea (One; who knows?).

- chaînes comprenant une noce/un mariage, comme AT 2019 Pif Paf Poltrie. Notons au passage que ce sous-groupe ne contient que deux contes-types.

- chaînes comprenant une mort, comme AT 202I Le Coq et la poule.

- chaînes comprenant le fait d'avaler un objet (les membres de la chaîne ne sont pas liés entre eux), comme AT 2025 The fleeing Pancake.

- chaînes contenant d'autres événements (actants non liés entre eux), comme AT 2030 The Old Woman and her Pig.

- chaînes avec actants interdépendants, comme AT 2015 The Goat who Would not Go Home.

- chaînes basées sur des actions, comme AT 2032 The Cock's Whiskers.

2) ATU (Uther 2004) a simplifié le système et distingue les sous-groupes suivants:

- chaînes basées sur des nombres, des objets, des animaux ou des noms (2000202O).

- chaînes comprenant une mort (2O2I-2024).

- chaînes comprenant le fait d'avaler (2025-2028).

- chaînes comprenant d'autres événements (2029-2075).

AT et ATU utilisent donc des critères de classification plutôt sémantiques.

3) Martti Haavio (I929: 64-92), quant à lui, distingue les sous-groupes suivants:

- randonnées par accumulation.

- randonnées par enchaînement.

- randonnées par une combinaison d'accumulation et d'enchaînement.

4) L'Association Croqu'Livre4 classe les randonnées en:

- chaînes par énumération; la plus simple. C'est une liste a + b + c, etc.

- chaînes par élimination. Le groupe perd ses membres un à un.

- chaînes par remplacement: a laisse sa place à b, b laisse sa place à c, etc.

- chaînes par emboîtement (poupées gigogne).

- toboggan: la chaîne se déroule dans le sens inverse.

Ces deux derniers systèmes utilisent donc des critères structuraux.

Ces catégories structurales font sûrement le bonheur des narratologues. Mais je vous avouerai que pour moi, le charme des randonnées est ailleurs. Assez fasti-

3. L'article de Taylor est une liste de changements qu'il suggère d'apporter à la première édition de l'Aarne-Thompson (I928) dont il espère une révision, qui sera effectuée en I96I.

4. Croqu'Livre. Centre régional de ressources en littérature jeunesse, Besançon, France. <www.croqulivre.asso.fr/selection/index.htm> 
dieuses à la lecture, elles prennent toute leur saveur dans une situation de contage, surtout si l'auditoire se prête au jeu et s'y implique.

D'ailleurs la plupart du temps, la cataloguisation des randonnées se fait facilement. En effet les variations se situent surtout au niveau de surface, celui des allomotifs, et le conte-type apparaît clairement: c'est la même histoire, avec des actants et des objets différents. Toutefois, il arrive que l'attribution d'un numéro ATU aux randonnées pose de sérieux problèmes, que je voudrais présenter avec l'exemple du conte-type La toute petite bonne femme (ATU 20I6 Wee Wee Woman) dont voici un exemple danois (ma traduction):

Il était une fois une toute petite bonne femme blanche, qui habitait dans une toute petite maison blanche, elle avait une toute petite vache blanche, et un tout petit chat blanc, et un tout petit seau blanc, et une toute petite jatte blanche, et une toute petite étagère blanche, et un tout petit bâton blanc. Un jour la petite bonne femme est allée traire sa toute petite vache blanche dans son tout petit seau blanc. Elle a mis le tout petit lait blanc dans une toute petite écuelle blanche qu'elle a posée sur la toute petite étagère blanche, et le tout petit chat blanc est venu et a bu le tout petit lait blanc. Alors la toute petite bonne femme s'est fâchée et a battu le tout petit chat blanc de son tout petit bâton blanc, et il s'est enfui au loin. (Tang Kristensen I896: I23-I24).

C'est une forme très simple de randonnée, un conte répétitif « avec action unique, sans dépendance réciproque des protagonistes ». Sa caractéristique principale est que l'adjectif « tout petit », « minuscule » s'applique à chaque être vivant, chaque objet et chaque action de l'histoire. Voici comment le catalogue ATU résume ce conte-type:

Once there was a teeny tiny woman who had a teeny tiny cow which she milked into a teeny tiny pail. A teeny tiny cat drank all the milk. The teeny tiny woman killed the cat (it died) and all the milk flowed back into the pail, etc. (ATU II: 5I6).

Il était une fois une toute petite bonne femme qui avait une toute petite vache qu'elle a traite dans un tout petit seau. Un tout petit chat a bu tout le lait. La toute petite bonne femme a tué son chat (il est mort) et tout le lait est remonté dans le seau, etc.

Nous ne connaissons pas l'origine de ce conte, et les versions écrites les plus anciennes ne remontent pas plus haut que le dix-neuvième siècle. Archer Taylor déclare que "The wee wee Woman n'est attestée qu'en Finlande, en Scandinavie et en en Angleterre ", et en conclut que ce conte est "probablement d'origine nordique » (Taylor I934-40: I76). Mais depuis I934, des versions ont été trouvées en Allemagne, en France, en Catalogne, en Italie, en Ukraine, en Biélorussie, en République tchèque et en Hongrie.

Les catalogues AT et ATU semblent utiliser la répétition du mot « tout petit » comme unique critère de distinction. Et dans la description qu'ils donnent de ce conte-type, le lait finit par rentrer dans le seau. En réalité, ce dénouement miraculeux ne se trouve que dans certaines versions. Dans les versions danoises ${ }^{5}$ (Tang Kristensen I896), la petite bonne femme bat son chat, qui meurt (no. 235), va

5. Cf. Tang Kristensen (I896: I2I-I24). 
retrouver sa mère (no. 236), s'enfuit (no. 23I), ou tout simplement crie « miaou! » (no. 232); et une version allemande se termine simplement par la petite bonne femme traitant son chat de « tout petit.$^{6}$

Les versions d'autres pays sont encore plus différentes. En Italie, ${ }^{7}$ la petite bonne femme est impliquée dans une intrigue tout autre. Sa toute petite poule pond un tout petit oeuf, dont elle fait une toute petite omelette, qu'elle met à refroidir sur le rebord de la fenêtre. Une toute petite mouche tombe dans l'omelette. La petite bonne femme porte plainte auprès de l'agent de police, qui lui suggère comme vengeance de frapper la mouche la prochaine fois qu'elle la verra. Une mouche se pose sur le nez de l'agent de police et la petite bonne femme la frappe, comme dans le conte facétieux ATU I586 The Man in Court for killing a Fly. Quant aux versions catalanes, ${ }^{8}$ ce sont des histoires tout à fait différentes. Une toute petite bonne femme qui a un tout petit chat se marie avec un tout petit bonhomme qui a un tout petit chien. Le tout petit chien, jaloux, tue le tout petit chat. « et mon histoire est finie ». Quant au conte anglais « The wee wee mannie », classé comme ATU 2016 à cause du motif initial, un tout petit bonhomme en train de traire une toute petite vache, il est en fait plus proche du conte ATU 20I5 "The Goat who would not go home ». Et une certaine version anglaise ${ }^{\mathrm{IO}}$ est la transformation créatrice du conte merveilleux ATU 366 The Man from the Gallows en un conte d'attrape.

Ainsi, le motif initial de la toute petite bonne femme introduit des intrigues extrêmement différentes. Il semble d'ailleurs exister des variations géographiques assez nettes, mais le matériel à notre disposition est trop mince pour nous permettre de parler véritablement d'écotypes.

Est-il légitime alors de caractériser un conte par un simple motif décoratif, qui est même inutile á l'intrigue?

\section{Randonnée et récit}

Le conte de La toute petite Bonne Femme ne peut pas être relié à quelque usage religieux ou rituel, comme cela a peut-être été le cas pour le conte de La Chèvre qui ne veut pas sortir du champ ; et il est peu probable qu'il ait été utilisé à des fins didactiques, comme le conte ATU 2009 L'Origine des échecs et ATU 2oro Ehod mi Yodea («One: who knows?»-Les douze Paroles de la vérité. C'est surtout un récit ludique. Ous ses formes les plus simples, c'est presque un conte d'attrape, qui frustre l'auditeur dans son attente d'un vrai récit complet. On peut y voir une parodie de conte, l'exploration facétieuse des limites de la narratologie. Jusqu'où peut-on pousser la simplicité d'un énoncé (situation initiale + action + situation finale), et la trivialité de ses détails, sans qu'il cesse d'être un récit? Il est vrai que d'autres cultures, celles des Amérindiens notamment étudiés par Alan Dundes notamment, ont parfois des récits très simples, où il n'y a parfois qu'une seule action

\footnotetext{
6. Cf. Kooi-Schuster (1994).

7. Cf. Imbriani (I877, XXXIX) et Papanti (I877: I5-I8, no. 3).

8. Cf. Alcover (1956, XV: I30-I32).

9. Cf. Jacobs (I894: I77-I79).

Io. Cf. Briggs « Klitzeklein » (I970: I24-I25).
} 
entre le manque et la résolution du manque. Mais dans la tradition européenne, les adultes attendent d'un conte un certain nombre d'épisodes.

Toutes les randonnées ne sont d'ailleurs pas des récits; Ainsi ATU 2035 The House that Jack built, pourtant rangé dans la classe des chaînes basées sur des actions. C'est une description, et les actions énumérées sont mentionnées au passé comme un attribut de chaque objet de la scène, et non comme le point focal de la narration.

L'AT et l'ATU ont des contes-types, qui sont de simples énumérations: 20I0A Les Dix Jours de Noël, et 20 Io I. Comment le fermier paie son valet. Il est d'ailleurs symptomatique que dans la tradition scandinave, ces deux récits qui n'en sont pas existent aussi sous forme de chansons énumératives.

\section{Randonnée et sens}

Sous des dehors facétieux, les randonnées traitent de thèmes très importants pour les sociétés rurales traditionnelles: la nécessité de l'entraide, du deuil collectif, etc. Pourtant, comme tous les "petits genres », les randonnées ont peu intéressé les chercheurs, sauf celles qu'ils ont cru pouvoir faire remonter á des liturgies juives, comme ATU 20Io Ehod mi Yodea (One; Who knows) (Kohuot I895) et ATU 20I5 La chèvre qui ne veut pas sortir du chou, que l'on a fait remonter à un récit talmudique. Pour les chercheurs du ige siècle, son antiquité lui donne des lettres de noblesse. Et sous sa forme chantée, c'est une des chansons que les instigateurs de la grande enquête Fortoul sur les « Poésies populaires de la France » (I857) demandent à leurs correspondants de collecter tout particulièrement, justement à cause de son grand âge et de son origine prétendument talmudique (Simonsen 2005-06).

Le folkloriste danois Bengt Holbek a écrit une petite étude sur Le Chat ventru extrêmement intéressante. C'est un conte très répandu au Danemark, où il en existe de nombreuses versions. Toutes ont la même structure, même si la nature des êtres avalés et parfois même de l'avaleur change beaucoup. Dans la version que je viens de vous conter, le grand plaisir du conte vient de l'absurdité et du caractère hétérogène des êtres avalés. Dans une version, Cela va même jusqu'au non-sens, puisque dans une version le chat rencontre successivement des êtres dont on ne sait ce qu'ils sont: Skohottentot, puis Skolinkelot, ce qui ne veut strictement rien dire, même pour un Danois. Ici, c'est le plaisir du charabia qui compte, la poésie pure dadaïste. Mais Holbek s'intéresse particulièrement aux versions dans lesquelles le chat avale successivement un paysan en train de labourer, un autre en train de herser, un troisième en train de semer, etc. Il en conclut que c'est une métaphore et un conte d'avertissement pour les communautés paysannes, toujours au bord de la disette; le chat (le paysan) a si faim qu'il dévore successivement tout ce qui doit assurer sa survie dans l'année qui vient (labourage, semaisons, etc.). En temps de famine, la tentation est grande de manger la semence qui doit assurer la récolte de l'année suivante, mais c'est une tentation à laquelle il faut résister, car si l'on y succombe, c'est à longue échéance la mort assurée. Ainsi, conclut Bengt Holbek, de menues variations de surface au niveau figuratif, celui des allomotifs, qui sont structurellement équivalents, peuvent changer complètement le sens d'une randonnée.

De nos jours, les randonnées sont surtout contées aux jeunes enfants, qui les apprécient beaucoup. Pour la psychologue Marie-Claire Bruley, elles constituent 
un repère organisateur de leur vie. Elles permettent de dénombrer, d'inventorier le réel, créent un ordre dans le chaos. De plus, les formules répétitives constituent un arrêt dans la narration, neutralisant ainsi la part d'inconnu qu'il y a dans tout récit. Et quand la petite vieille et tout ceux que le chat a avalés sortent ensemble du ventre du chat, l'enfant retrouve en un instant l'ensemble des séquences qui ont construit progressivement l'histoire, ce qui constitue une initiation à la mâ̂trise du récit.

\section{Randonnée et facétie}

Le contenu de la randonnée est souvent facétieux, suivant les deux principaux procédés du facétieux populaire: l'hyperbole, exagération monumentale, et l'incongru, la mise sur le même plan d'éléments tout à fait hétérogènes cf. ATU 2028 The devouring Animal that Was Cut Open ou ATU 2Io I How the Rich Man paid his Servant. Dans ce dernier cas, le rire est créé par l'absurde, mais c'est aussi un rire satirique: la démonstration par l'absurde que les valets reçoivent des salaires de misère.

On le sait, les enfants adorent les répétitions, et la littérature orale adore les inventaires, surtout s'ils sont incongrus, c'est-à-dire s'ils alignent à la queue leu des éléments disparates. Pensons aux fatrasies du Moyen-Âge, pensons à Rabelais et au catalogue de la librairie Saint-Victor ; ${ }^{\text {II }}$ aux jeux de Gargantua, ${ }^{\text {I2 }}$ à l'épisode du torche-cul; ${ }^{13}$ pensons à Prévert et son raton-laveur, ${ }^{14}$ etc.

\section{Randonnée et virelangue}

Par ailleurs, les randonnées contiennent un fort élément de virelangue (« tonguetwister » en anglais). Ceci semble particulièrement vrai des versions catalanes. Mais d'une façon générale, toute randonnée quand elle est dite à haute voix doit être dite d'un seul souffle; ce n'est pas la logique de l'histoire qui détermine la longueur de la chaîne, c'est la longueur du souffle, et donc l'agilité verbale du conteur. Si le conteur reprend son souffle avant la fin de la chaîne, l'effet est coupée. Les randonnées sont très conscientes de cet aspect "virelangue ", puisque certaines d'entre elles utilisent des onomatopées-cris d'animaux, ou bien même des mots inventés: Voici par exemple une version française de la randonnée ATU 2022, où les animaux et les objets prennent successivement le deuil de la petite poule morte:
«Si la rate n'est pas morte, je ne peux plus miauler », dit le chat.
« Ni moi fenêtrer», dit la fenêtre.
«Ni moi banquer», dit le banc.
«Ni moi marmiter», dit la marmite.
« Ni moi crémaillèrer », dit la crémaillère.

\footnotetext{
II. Rabelais, Pantagruel : VII.

I2. Rabelais, Gargantua: XXII

I3. Rabelais, Gargantua:XIII

I4. Prévert: «Inventaire » (I946).
} 
« Ni moi quenouiller », dit la quenouille.

Le rat et la rate mangèrent la bouillie de bon appétit.

(Bladé I886, vol. 3:235-237).

Dans la pratique, surtout avec des enfants, le conteur cherche á impliquer l'auditoire et à lui faire dire le refrain répétitif, ce qu'il fait d'ailleurs avec plus ou moins d'agilité. Plus l'auditoire s'en tire mal et plus le plaisir de tous est grand, du moins avec des adultes. Avec les enfants, c'est plutôt le plaisir de dire ensemble un texte rythmé qui l'emporte.

Par ailleurs, le conteur de randonnées utilise non seulement sa voix, mais aussi son corps. La conteuse Hélène Loup, qui affectionne ce type de contes et leur a consacré un ouvrage remarquable et tout à fait novateur, donne une description précise des gestes qu'elle associe au contage de Pou et puce.

En effet, plus que tous les autres types de contes, les randonnées prennent tout leur sens dans une performance orale, où l'interaction entre conteur et auditoire est la raison d'être du récit.

La spécificité de l'art du conte... est de permettre une interaction immédiate entre conteur(s) et auditeurs. La randonnée est, avec la devinette la forme la plus achevée, la plus ludique de cet art du partage. (Loup 2000: 22-26).

\section{Références bibliographiques}

Aarne, Antti ; Stith Thompson (I96I) : The Types of the Folktale. A Classification and Bibliography. Folklore Fellows' Communications I84. Helsinki: Suomalainen Tiedeakatemia.

Alcover, Antoni M. (I956): Aplec de Rondaies Mallorquines d'En Jordi des Recó. Vol. XV. Palma.

BlADÉ, Jean-François (I886): Contes de Gascogne. Paris: Maisonneuve.

BRIGGS, Katharina (I970) : "Klitzeklein ». Englische Volksmärchen. Eugen Diederichs Verlag.

BRuley, Marie-Claire (I988) : Enfantines et berceuses. Paris : Ecole des loisirs. $<$ http://www.didier-jeunesse.com>

HAAVIO, Martti (I929) : Kettenmärchenstudien. I. Folklore Fellows' Communication 88. Helsinki: Suomalainen Tiedeakatemia.

HolbeK, Bengt (1978) : " Den bugede kat: Forsøg på en strukturanalyse ». Folk og Kultur (1978) : 59-73. [English translation: «The big-bellied Cat ». In Alan Dundes (ed.) (I978): Varia Folclorica. The Hague-Paris: Mouton, p. 57-70.]

IMBRIANI, Vittorio (1877): La Novellaja Fiorentina. In Livorno: Coi tipi di F. Vigo.

JaCOBS, Joseph (I894): More English Fairy Tales. London: D. Nutt.

Kohut, G. A. (I895) : "Le had gadya et les chansons similaires ». Revue des études juives 3I, (I895): 240-246.

Kooi, J. van der; Theo SCHUSTER (I994) : Der Grossherzog und die Marktfrau. Märchen und Schwänke aus dem Oldenburger Land. Leer: Schuster. 
Loup, Hélène (200o) : Le Jeu de la Répétition dans les Contes ou Comment Dire et Redire sans se Répéter. L'espace du conte. Paris: Edisud.

PAPANTI, Giovanni (I877): Novelline popolari Livornesi. Livorno: F. Vigo.

Prévert, Jacques (I946): Paroles. Le Point du Jour.

Simonsen, Michèle (2005-06) : " Poesies populares de la France $=$ The fortoul collection of french folksongs (I853-I855) ». Estudos de literatura oral n. ${ }^{\circ}$ II-I2 (2005-06): 253-268.

TAYLOR, Archer (I933) : «A Classification of Formula Tales ». Journal of American Folklore, vol. 46, n. ${ }^{\circ}$ I79 (January-March I933): 77-88.

- (I934) : "Formelmärchen ». Handwörterbuch des deutschen Märchen II (I934): 77-88.

UTHER, Hans-Jörg (2004): The Types of International Folktales. A Classification and Bibliography. Vol. II. Folklore Fellows' Communications 285. Helsinki: Suomalainen Tiedeakatemia.

WiEnKer-Piepho, Sabine (I993) : « Kettenmärchen ». Enzyklopädie des Märchens. Berlin/New York: Walter de Gruyter, vol. 7 (4/5): II94-I2OI. 